\title{
Orbital motions as gradiometers for post-Newtonian tidal effects
}

\author{
Lorenzo lorio* \\ Ministero dell'Istruzione, dell'Università e della Ricerca, Istruzione, Bari, Italy
}

\section{Edited by:}

Martin Anthony Hendry, University

of Glasgow, UK

Reviewed by:

Zurab Silagadze, Novosibirsk State University, Russia

Lee Samuel Finn, The Pennsylvania

State University, USA

*Correspondence:

Lorenzo lorio, Ministero

dell'Istruzione, dell'Università e della

Ricerca, Istruzione, Viale Unità di

Italia 68, Bari 70125, Italy

e-mail: lorenzo.iorio@libero.it
The direct long-term changes occurring in the orbital dynamics of a local gravitationally bound binary system $S$ due to the post-Newtonian tidal acceleration caused by an external massive source are investigated. A class of systems made of a test particle $m$ rapidly orbiting with orbital frequency $n_{\mathrm{b}}$ an astronomical body of mass $M$ which, in turn, slowly revolves around a distant object of mass $M^{\prime}$ with orbital frequency $n_{\mathrm{b}}{ }^{\prime} \ll n_{\mathrm{b}}$ is considered. The characteristic frequencies of the non-Keplerian orbital variations of $m$ and of $M$ itself are assumed to be negligible with respect to both $n_{\mathrm{b}}$ and $n_{\mathrm{b}}{ }^{\prime}$. General expressions for the resulting Newtonian and post-Newtonian tidal orbital shifts of $m$ are obtained. The future missions BepiColombo and JUICE to Mercury and Ganymede, respectively, are considered in view of a possible detection. The largest effects, of the order of $\approx 0.1-0.5$ milliarcseconds per year (mas $\mathrm{yr}^{-1}$ ), occur for the Ganymede orbiter of the JUICE mission. Although future improvements in spacecraft tracking and orbit determination might, perhaps, reach the required sensitivity, the systematic bias represented by the other known orbital perturbations of both Newtonian and post-Newtonian origin would be overwhelming. The realization of a dedicated artificial mini-planetary system to be carried onboard and Earth-orbiting spacecraft is considered as well. Post-Newtonian tidal precessions as large as $\approx 1-10^{2}$ mas $\mathrm{yr}^{-1}$ could be obtained, but the quite larger Newtonian tidal effects would be a major source of systematic bias because of the present-day percent uncertainty in the product of the Earth's mass times the Newtonian gravitational parameter.

Keywords: general relativity and gravitation, experimental studies of gravity, experimental tests of gravitational theories, celestial mechanics, spacecraft

\section{INTRODUCTION}

Gravitation is one of the known fundamental interactions of physics, and the General Theory of Relativity (GTR) is, at present, its best theoretical description (Will, 2009). As such, GTR is one of the pillars of our knowledge of Nature; intense experimental and observational scrutiny is required not only to gain an everincreasing confidence about it, but also to explore the borders of the realm of its validity at different scales. To this aim, a variety of different theoretical, experimental and observational approaches are required to extend the frontiers of our knowledge of the gravitational phenomena. Are there some founded hopes to testing newly predicted gravitational effects in the near future in some suitable astronomical and astrophysical laboratories? What are the possibilities opened up by forthcoming space-based missions? The present paper will try to address these questions by looking at certain effects that the components of the Riemann spacetime curvature tensor are expected to induce on local systems according to GTR.

The internal dynamics of a gravitationally bound binary system immersed in the external gravitational field of a massive rotating body is tidally affected at both the Newtonian and the post-Newtonian level (Mashhoon, 1977; Chicone and Mashhoon, 2002, 2006; Kopeikin et al., 2011). In this paper, we will look in detail at some of the post-Newtonian orbital effects of tidal origin arising in the relative motion of a restricted two-body system, and at the possibility of detecting them in either natural or artificial space-based scenarios. Special cases widely treated in the literature are the post-Newtonian tidal effects of the rotating Sun's field in the Earth-Moon system (Braginsky and Polnarev, 1980; Mashhoon and Theiss, 1982, 1986; Gill et al., 1989; Mashhoon and Theiss, 1991, 2001) and of the spinning Earth itself in arrays of spaceborne artificial gradiometers (Mashhoon and Theiss, 1982; Theiss, 1985; Mashhoon et al., 1989; Paik, 1989; Theiss, 1992; Paik, 2008; Li et al., 2014). Our calculation will have a broad range of validity. Indeed, while, on the one hand, certain assumptions on the characteristic orbital frequencies of the three-body system considered will be necessarily made, on the other hand, we will remove the limitations existing in the literature (Chicone and Mashhoon, 2006) on either the orientation of the spin axes of the external objects and on the orbital configurations of the moving bodies.

The paper is organized as follows. In Section 2, the long-term rates of change of the orbital parameters of the test particle of the restricted two-body system are calculated by keeping the elements of a generic tidal matrix constant over the orbital period of the particle around its primary. In Section 3, the direct orbital 
effects due to both the gravitoelectric and the gravitomagnetic tidal matrices are obtained by averaging their elements over the orbital period of the motion around the distant body. Section 4 is devoted to exploring some experimental possibilities offered by forthcoming spacecraft-based missions to astronomical bodies. Section 5 summarizes our findings.

\section{THE LONG-TERM ORBITAL RATES OF CHANGE AVERAGED OVER $P_{\mathrm{B}}$}

Let us consider an isolated rotating body of mass $M^{\prime}$, equatorial radius $R^{\prime}$ and proper angular momentum $J^{\prime}$ at rest in some parameterized post-Newtonian coordinate system $\mathcal{K}^{\prime}$ whose spatial axes point to distant stars; as such, $\mathcal{K}^{\prime}$ is kinematically and dynamically non-rotating (Brumberg and Kopeikin, 1989). Let a local gravitationally bound system $S$ move geodesically around $M^{\prime}$; $S$ is assumed to be made of a body of mass $M$, equatorial radius $R$ and proper angular momentum $J$, and of a test particle of mass $m$ revolving about $M$ itself. For the sake of simplicity, we will assume $m \ll M \ll M^{\prime}$; examples of such scenarios are spacecraft like MESSENGER (Solomon et al., 2007) and the forthcoming BepiColombo (Benkhoff et al., 2010) to orbit Mercury in the field of the Sun as well as the future JUICE mission (Grasset et al., 2013) to orbit Ganymede and to study the Jovian system. To the Newtonian level, the spatial trajectory of $S$ about $M^{\prime}$ can be parameterized in terms of the usual Keplerian orbital elements. They are the semimajor axis $a^{\prime}$, the eccentricity $e^{\prime}$, the inclination $I^{\prime}$ to the reference $\left\{x^{\prime}, y^{\prime}\right\}$ plane of $\mathcal{K}^{\prime}$, the longitude of the ascending node $\Omega^{\prime}$, and the argument of pericenter $\omega^{\prime} ; n_{\mathrm{b}}{ }^{\prime}=\sqrt{G M^{\prime} a^{\prime-3}}$ is the Keplerian orbital frequency, where $G$ is the Newtonian constant of gravitation. In general, such orbital parameters do not stay constant because of the well known Newtonian and postNewtonian departures from spherical symmetry of the field of $M^{\prime}$. They induce the post-Newtonian Einstein (gravitoelectric) (Einstein, 1915; Nobili and Will, 1986) and Lense-Thirring (gravitomagnetic) (Lense and Thirring, 1918) orbital precessions as well as the classical ones due to the oblateness $J_{2}{ }^{\prime}$ of $M^{\prime}$ and, possibly, to its other multipoles of higher order (Bertotti et al., 2003).

Let a local inertial frame $\mathcal{K}$, attached to $M$, be parallel transported along the geodesic worldline of $M$ through the spacetime of $M^{\prime}$ (Fermi, 1922; Levi-Civita, 1926; Synge, 1927). As such, the spatial axes of $\mathcal{K}$ change naturally their orientation with respect to the fixed "Copernican" spatial axes of $\mathcal{K}$ ' because of the geodesic motion of $\mathcal{K}$ itself through the external spacetime deformed by $M^{\prime}$ and $\boldsymbol{J}^{\prime}$. As such, $\mathcal{K}$ is also said to be kinematically rotating because of the resulting de Sitter-Fokker (gravitoelectric) (de Sitter, 1916; Fokker, 1921) and Pugh-Schiff (gravitomagnetic) precessions $^{1}$ of its axes with respect to those of $\mathcal{K}^{\prime}$, but it is dynamically nonrotating because of the absence of Coriolis and centrifugal inertial forces (Brumberg and Kopeikin, 1989). To the Newtonian level, also the motion of $m$ about $M$ will be parameterized in terms of a set of Keplerian orbital elements $a, e, I, \Omega, \omega$ in such a way that $n_{\mathrm{b}}=\sqrt{G M a^{-3}}$ denotes its Keplerian orbital frequency. Usually, the frequencies of the de Sitter-Fokker and

${ }^{1} \mathrm{~A}$ comoving coordinate system is said to be kinematically nonrotating if it is corrected for the post-Newtonian precessions of its axes.
Pugh-Schiff precessions are quite smaller than both $n_{\mathrm{b}}{ }^{\prime}$ and $n_{\mathrm{b}}$; for a critical discussion tidal phenomena occurring in the SunEarth-Moon system over timescales comparable to or larger than the de Sitter-Fokker and Pugh-Schiff ones, see (Gill et al., 1989; Mashhoon and Theiss, 1991). Thus, we can safely assume $\mathcal{K}$ as kinematically nonrotating over timescales comparable to the orbital periods $P_{\mathrm{b}}=2 \pi n_{\mathrm{b}}^{-1}$ and $P_{\mathrm{b}}^{\prime}=2 \pi n_{\mathrm{b}}{ }^{\prime-1}$ of the three-body system considered. As a further assumption, we will consider the local motion of $m$ about $M$ much faster than the one of $S$ itself around $M^{\prime}$, i.e., $n_{\mathrm{b}}{ }^{\prime} \ll n_{\mathrm{b}}$. In general, the internal dynamics of $S$ is not purely Keplerian because of possible departures from sphericity of $M$ and of the post-Newtonian components of the field of $M$. As such, the orbit of $m$ with respect to $M$ undergoes the well-known Newtonian and post-Newtonian orbital precessions. As it occurs in the systems considered here, the timescales of such changes are quite longer than the orbital periods $P_{\mathrm{b}}, P_{\mathrm{b}}^{\prime}$, i.e., $\dot{\Psi} \ll n_{\mathrm{b}}{ }^{\prime}$, where $\Psi$ denotes a generic precessing osculating Keplerian orbital element of $m$.

At both the Newtonian and the post-Newtonian level, the internal dynamics of $S$ is locally affected also by tidal effects due to its motion through the external deformed spacetime of $M^{\prime}$. The tidal acceleration experienced by $m$ is of the form (Mashhoon et al., 1989)

$$
A_{\mathrm{tid}}=-\mathbf{K} r
$$

where the elements of the tidal matrix $\mathbf{K}$

$$
\mathrm{K}_{i j}=\mathcal{R}_{0 i 0 j}, i, j=1,2,3
$$

are the tetrad components of the curvature Riemann tensor evaluated onto the geodesic of the observer in $\mathcal{K}$, and have dimensions of $\mathrm{T}^{-2}$. It is

$$
\mathbf{K}=\mathbf{K}^{(\mathrm{N})}+\mathbf{K}^{(\mathrm{GE})}+\mathbf{K}^{(\mathrm{GM})},
$$

with the Newtonian $(\mathrm{N})$, gravitoelectric (GE) and gravitomagnetic (GM) tidal matrices given by (Mashhoon et al., 1989)

$$
\begin{aligned}
& \mathrm{K}_{i j}^{(\mathrm{N})}=\frac{G M^{\prime}}{r^{\prime 3}}\left(\delta_{i j}-3{\hat{r^{\prime}}}_{{ }^{\prime}}{\hat{r^{\prime}}}_{j}\right), \\
& \mathrm{K}_{i j}^{(\mathrm{GE})}=-\frac{G^{2} M^{\prime 2}}{c^{2}{r^{\prime}}^{4}}\left(3 \delta_{i j}-9{\hat{r^{\prime}}}_{{ }^{\prime}}{\hat{r^{\prime}}}_{j}\right) \\
& +\frac{G M^{\prime}}{c^{2} r^{\prime 3}}\left\{3\left[v^{\prime 2} \delta_{i j}-v_{i}^{\prime} v^{\prime}{ }_{j}+3\left(\boldsymbol{v}^{\prime} \cdot \hat{\boldsymbol{r}}^{\prime}\right){\hat{r^{\prime}}}_{(i} v^{\prime}{ }_{j)}\right]\right. \\
& \left.-3\left(\boldsymbol{v}^{\prime} \cdot \hat{\boldsymbol{r}}^{\prime}\right)^{2} \delta_{i j}-6{\hat{r^{\prime}}}_{i}{\hat{r^{\prime}}}_{j} v^{\prime 2}\right\} \\
& \mathrm{K}_{i j}^{(\mathrm{GM})}=-\frac{6 G J^{\prime}}{c^{2} r^{\prime}}\left\{3\left(\boldsymbol{v}^{\prime} \times \hat{\boldsymbol{k}}^{\prime}\right)_{(i}{\hat{r^{\prime}}}_{j)}+\left(\hat{\boldsymbol{r}}^{\prime} \times \hat{\boldsymbol{k}}^{\prime}\right)_{(i} v_{j}^{\prime}\right. \\
& +\hat{\boldsymbol{r}^{\prime}} \cdot\left(\boldsymbol{v}^{\prime} \times \hat{\boldsymbol{k}^{\prime}}\right)\left(\delta_{i j}-5{\hat{r^{\prime}}}_{{ }^{\prime}}{\hat{r^{\prime}}}_{j}\right) \\
& \left.-5\left(\hat{\boldsymbol{r}^{\prime}} \cdot \boldsymbol{v}^{\prime}\right)\left(\hat{\boldsymbol{r}}^{\prime} \times \hat{\boldsymbol{k}}^{\prime}\right)_{(i}{\hat{r^{\prime}}}_{j)}\right\} \text {. }
\end{aligned}
$$

In Equations (4-6), which are symmetric and traceless, $c$ is the speed of light in vacuum, $\hat{\boldsymbol{r}^{\prime}}=\boldsymbol{r}^{\prime} / \boldsymbol{r}^{\prime}$ is the versor of the position 
vector $\boldsymbol{r}^{\prime}$ from $M^{\prime}$ to $M, \boldsymbol{v}^{\prime}$ is the velocity vector of $M$ with respect to $M^{\prime}, \delta_{i j}$ is the Kronecker symbol, $\hat{\boldsymbol{k}}^{\prime}$ is the unit vector of the spin axis of $M^{\prime}$, the symbols $\cdot$ and $\mathbf{x}$ denote the usual scalar and cross products among vectors, and parentheses around indices denote symmetrization.

The tidal acceleration of Equation (1) can be considered as a small perturbation $A_{\text {pert }}$ of the Newtonian monopole of $M$. As such, its impact on the orbital dynamics of $m$ can be treated perturbatively with standard techniques. By recalling the condition $n_{\mathrm{b}}{ }^{\prime} \ll n_{\mathrm{b}}$, the elements of the tidal matrix $\mathbf{K}$ can be considered as constant over an orbital period $P_{\mathrm{b}}$. Thus, by evaluating the right-hand-sides of the Gauss equations (Burns, 1976)

$$
\begin{aligned}
\frac{d a}{d t} & =\frac{2}{n_{\mathrm{b}} \sqrt{1-e^{2}}}\left(e A_{R} \sin f+\frac{p}{r} A_{T}\right), \\
\frac{d e}{d t} & =\frac{\sqrt{1-e^{2}}}{n_{\mathrm{b}} a}\left\{A_{R} \sin f+A_{T}\left[\cos f+\frac{1}{e}\left(1-\frac{r}{a}\right)\right]\right\}, \\
\frac{d I}{d t} & =\frac{\cos (\omega+f)}{n_{\mathrm{b}} a \sqrt{1-e^{2}}}\left(\frac{r}{a}\right) A_{N}, \\
\frac{d \Omega}{d t} & =\frac{\sin (\omega+f)}{n_{\mathrm{b}} a \sin I \sqrt{1-e^{2}}}\left(\frac{r}{a}\right) A_{N}, \\
\frac{d \omega}{d t}+\cos I \frac{d \Omega}{d t} & =\frac{\sqrt{1-e^{2}}}{n_{\mathrm{b}} a e}\left[-A_{R} \cos f+A_{T}\left(1+\frac{r}{p}\right) \sin f\right] .(11)
\end{aligned}
$$

onto the unperturbed Keplerian ellipse

$$
r=\frac{p}{1+e \cos f}
$$

where $p=a\left(1-e^{2}\right)$ is the semilatus rectum and $A_{R}, A_{T}, A_{N}$ are the radial, transverse and out-of-plane components of Equation (1), the orbital variations of $m$ averaged over $P_{\mathrm{b}}$ can be calculated. To this aim, let us note that, in principle, the average should be made by means of (Brumberg, 1991; Will, 2014)

$$
\frac{d f}{d t}=n_{\mathrm{b}}\left(\frac{a}{r}\right)^{2} \sqrt{1-e^{2}}-\left(\frac{d \omega}{d t}+\cos I \frac{d \Omega}{d t}\right),
$$

where $t$ is the proper time along the observer's geodesic, $f$ is the true anomaly, and $\dot{\Omega}, \dot{\omega}$ are to be intended as the instantaneous, non-averaged precessions of the node and the pericenter (Brumberg, 1991). Indeed, $f$ is reckoned from the pericenter position, which, in general, changes because of possible variations of $\Omega$ and $\omega$ due to the non-Keplerian Newtonian and post-Newtonian effects within $S$. As such, the instantaneous expressions for $\dot{\Omega}$ and $\dot{\omega}$ in Equation (13) should be taken from Equations $(10,11)$ themselves evaluated for the specific Newtonian and post-Newtonian perturbations (Brumberg, 1991). However, by limiting ourselves just to the order $\mathcal{O}\left(c^{-2}\right)$, and by neglecting small mixed terms of order $\mathcal{O}\left(J_{2} c^{-2}\right)$ arising from the interplay between the external tidal and the local Newtonian perturbations due to $J_{2}$, the approximate expression

$$
\frac{d f}{d t}=n_{\mathrm{b}}\left(\frac{a}{r}\right)^{2} \sqrt{1-e^{2}}
$$

can be used by integrating over $f$ between 0 and $2 \pi$. By using the following Keplerian expressions for the position $\boldsymbol{r}$

$$
\begin{aligned}
& x=r[\cos \Omega \cos (f+\omega)-\cos I \sin \Omega \sin (f+\omega)], \\
& y=r[\sin \Omega \cos (f+\omega)+\cos I \cos \Omega \sin (f+\omega)], \\
& z=r[\sin I \sin (f+\omega)],
\end{aligned}
$$

and the velocity $v$

$$
\begin{aligned}
& a n_{\mathrm{b}}\{\cos I \sin \Omega[e \cos \omega+\cos (f+\omega)] \\
& v_{x}=-\frac{+\cos \Omega[e \sin \omega+\sin (f+\omega)]\}}{\sqrt{1-e^{2}}}, \\
& a n_{\mathrm{b}}\{\cos I \cos \Omega[e \cos \omega+\cos (f+\omega)] \\
& v_{y}=\frac{-\sin \Omega[e \sin \omega+\sin (f+\omega)]\}}{\sqrt{1-e^{2}}}, \\
& v_{z}=\frac{a n_{\mathrm{b}} \sin I[e \cos \omega+\cos (f+\omega)]}{\sqrt{1-e^{2}}},
\end{aligned}
$$

it is possible to compute from Equations (15-20) the unit vector $\hat{L}$ along the orbital angular momentum as

$$
\hat{L}=\frac{r \times v}{|r \times v|}
$$

Then, the radial, transverse and normal components of Equation (1) turn out to be

$$
\begin{aligned}
A_{R}= & \boldsymbol{A}_{\mathrm{tid}} \cdot \hat{\boldsymbol{r}}=\frac{\mathrm{Ka}\left(1-e^{2}\right)}{1+e \cos f} \\
& \cdot\{[\cos I \sin (f+\omega) \sin \Omega-\cos (f+\omega) \cos \Omega] \\
& \cdot\left[\operatorname { s i n } f \left(K_{21} \cos I \cos \omega \cos \Omega+\left(K_{22}+K_{33}\right) \sin \omega \cos \Omega\right.\right. \\
& \left.+K_{31} \cos \omega \sin I\right)+\cos f\left(K_{11} \cos \omega \cos \Omega\right. \\
& \left.+\left(K_{21} \cos I \cos \Omega+K_{31} \sin I\right) \sin \omega\right)+\left(K_{21} \cos (f+\omega)\right. \\
& \left.\left.+\left(K_{22}+K_{33}\right) \cos I \sin (f+\omega)\right) \sin \Omega\right] \\
& +(\cos I \cos \Omega \sin (f+\omega)+\cos (f+\omega) \sin \Omega) \\
& \cdot\left(\cos (f+\omega)\left(K_{21} \cos \Omega+K_{22} \sin \Omega\right)\right. \\
& \left.+\sin (f+\omega)\left(K_{32} \sin I+\cos I\left(K_{22} \cos \Omega-K_{21} \sin \Omega\right)\right)\right) \\
& +\sin I \sin (f+\omega)\left(\cos (f+\omega)\left(K_{31} \cos \Omega+K_{32} \sin \Omega\right)\right. \\
& \left.\left.+\sin (f+\omega)\left(K_{33} \sin I+\cos I\left(K_{32} \cos \Omega-K_{31} \sin \Omega\right)\right)\right)\right\},
\end{aligned}
$$

$$
\begin{aligned}
A_{T}= & A_{\mathrm{tid}} \cdot(\hat{L} \times \hat{r})=-\frac{\mathrm{K} a\left(1-e^{2}\right)}{4(1+e \cos f)}\left\{\left(-K_{33}+\left(2 K_{22}+K_{33}\right)\right.\right. \\
& \left.\cdot \cos 2 \Omega-2 K_{21} \sin 2 \Omega\right) \cos ^{2} I \sin (2 f+2 \omega) \\
& +2\left(2 \sin I\left(K_{32} \cos \Omega-K_{31} \sin \Omega\right) \sin (2 f+2 \omega)\right.
\end{aligned}
$$




$$
\begin{aligned}
& \left.+\left[2 K_{21} \cos 2 \Omega+\left(2 K_{22}+K_{33}\right) \sin 2 \Omega\right]\right) \cos I \cos (2 f+2 \omega) \\
& +4 \sin I\left(K_{31} \cos \Omega+K_{32} \sin \Omega\right) \cos (2 f+2 \omega) \\
& +\left[(2-\cos 2 I) K_{33}+\left(2 K_{22}+K_{33}\right) \cos 2 \Omega\right. \\
& \left.\left.-2 K_{21} \sin 2 \Omega\right] \sin (2 f+2 \omega)\right\}, \\
A_{N}= & A_{\text {tid }} \cdot \hat{L}=-\frac{K a\left(1-e^{2}\right)}{4(1+e \cos f)}\left\{4 \cos I\left(K_{31} \cos \Omega+K_{32} \sin \Omega\right)\right. \\
& \cdot \cos (f+\omega)+2 \sin I\left[-2 K_{21} \cos 2 \Omega-\left(2 K_{22}+K_{33}\right) \sin 2 \Omega\right] \\
& \cdot \cos (f+\omega)+4 \cos 2 I\left(K_{32} \cos \Omega-K_{31} \sin \Omega\right) \sin (f+\omega) \\
& +\sin 2 I\left[3 K_{33}-\left(2 K_{22}+K_{33}\right) \cos 2 \Omega+2 K_{21} \sin 2 \Omega\right] \\
& \cdot \sin (f+\omega)\},
\end{aligned}
$$

where $\mathrm{K}$ is the dimensional scaling factor of the tidal matrix considered having dimensions of $\mathrm{T}^{-2}$, while the dimensionless coefficients $K_{i j}, i, j=1,2,3$ depend only on the orbital parameters of $M$ and on the orientation of $\hat{\boldsymbol{k}}^{\prime}$. Thus, by inserting Equations (22-24) in the right-hand-sides of Equations (7-11) and averaging them over $P_{\mathrm{b}}$ with Equation (14), one finally has

$$
\begin{aligned}
& \left\langle\frac{d a}{d t}\right\rangle_{P_{\mathrm{b}}}=0 \\
& \left\langle\frac{d e}{d t}\right\rangle_{P_{\mathrm{b}}}=\frac{5 \mathrm{Ke} \sqrt{1-e^{2}}}{8 n_{\mathrm{b}}}\left\{-2 \sin 2 \omega \sin \Omega\left(2 K_{21} \cos \Omega+K_{33} \sin \Omega\right)\right. \\
& +\cos ^{2} I+2\left[2 \sin I \sin 2 \omega\left(K_{32} \cos \Omega-K_{31} \sin \Omega\right)\right. \\
& \left.+\cos 2 \omega\left(2 K_{21} \cos 2 \Omega+\left(2 K_{22}+K_{33}\right) \sin 2 \Omega\right)\right] \cos I \\
& +4 \cos 2 \omega \sin I\left(K_{31} \cos \Omega+K_{32} \sin \Omega\right) \\
& +\sin 2 \omega\left[K_{22}(\cos 2 I+3) \cos 2 \Omega\right. \\
& \left.\left.+K_{33}(-\cos 2 I+\cos 2 \Omega+2)-2 K_{21} \sin 2 \Omega\right]\right\}, \\
& \left\langle\frac{d I}{d t}\right\rangle_{P_{\mathrm{b}}}=-\frac{\mathrm{K}}{8 n_{\mathrm{b}} \sqrt{1-e^{2}}}\left\{10 e^{2} \cos 2 I \sin 2 \omega\left(K_{32} \cos \Omega-K_{31} \sin \Omega\right)\right. \\
& -\frac{5}{2} e^{2} \sin 2 I \sin 2 \omega\left[-3 K_{33}+\left(2 K_{22}+K_{33}\right) \cos 2 \Omega\right. \\
& \left.-2 K_{21} \sin 2 \Omega\right]+2 \cos I\left(5 e^{2} \cos 2 \omega+3 e^{2}+2\right) \\
& \text {. }\left(K_{31} \cos \Omega+K_{32} \sin \Omega\right)-\left(5 e^{2} \cos 2 \omega+3 e^{2}+2\right) \sin I \\
& \text { - } \left.\left[2 K_{21} \cos 2 \Omega+\left(2 K_{22}+K_{33}\right) \sin 2 \Omega\right]\right\} \text {, } \\
& \left\langle\frac{d \Omega}{d t}\right\rangle_{P_{\mathrm{b}}}=-\frac{\mathrm{K} \csc I}{16 n_{\mathrm{b}} \sqrt{1-e^{2}}}\left\{2 0 e ^ { 2 } \operatorname { s i n } 2 \omega \left(\cos I\left(K_{31} \cos \Omega+K_{32} \sin \Omega\right)\right.\right. \\
& \left.-\sin I\left(K_{21} \cos 2 \Omega+\left(2 K_{22}+K_{33}\right) \cos \Omega \sin \Omega\right)\right) \\
& +4 \cos 2 I\left(5 e^{2} \cos 2 \omega-3 e^{2}-2\right)\left(K_{31} \sin \Omega-K_{32} \cos \Omega\right) \\
& +\left(5 e^{2} \cos 2 \omega-3 e^{2}-2\right) \sin 2 I\left[-3 K_{33}+\left(2 K_{22}+K_{33}\right)\right. \\
& \left.\left.\cdot \cos 2 \Omega-2 K_{21} \sin 2 \Omega\right]\right\} \text {, } \\
& \left\langle\frac{d \omega}{d t}\right\rangle_{P_{\mathrm{b}}}+\cos I\left\langle\frac{d \Omega}{d t}\right\rangle_{P_{\mathrm{b}}} \\
& =\frac{\mathrm{K} \sqrt{1-e^{2}}}{16 n_{\mathrm{b}}}\left\{-40 \sin I \sin 2 \omega\left(K_{31} \cos \Omega+K_{32} \sin \Omega\right)\right.
\end{aligned}
$$

$$
\begin{aligned}
& +4(5 \cos 2 \omega-3) \sin 2 I\left(K_{32} \cos \Omega-K_{31} \sin \Omega\right) \\
& -20 \cos I \sin 2 \omega\left[2 K_{21} \cos 2 \Omega+\left(2 K_{22}+K_{33}\right) \sin 2 \Omega\right] \\
& +\cos 2 I(5 \cos 2 \omega-3)\left[-3 K_{33}+\left(2 K_{22}+K_{33}\right) \cos 2 \Omega\right. \\
& \left.-2 K_{21} \sin 2 \Omega\right]+3(5 \cos 2 \omega+1)\left[K_{33}+\left(2 K_{22}+K_{33}\right)\right. \\
& \left.\left.\cdot \cos 2 \Omega-2 K_{21} \sin 2 \Omega\right]\right\}
\end{aligned}
$$

The long-term rates of Equations (25-29) are valid for any symmetric and traceless tidal-type perturbation of the form of Equation (1) whose coefficients can be considered as constant over the characteristic orbital frequency $n_{\mathrm{b}}$ of the local binary system considered. As such, Equations (25-29) are not limited just to Equations $(5,6)$. Moreover, Equations (25-29) hold for a general orbital configuration of the test particle $m$ since no a priori simplifying assumptions concerning its eccentricity and inclination were made. As shown by Equation (26), the eccentricity of a circular orbit is not affected by a tidal-type perturbation.

\section{THE LONG-TERM ORBITAL RATES OF CHANGE AVERAGED OVER $\boldsymbol{P}_{b}^{\prime}$}

In general, Equations (25-29) may not be regarded as truly secular rates over timescales arbitrarily long because of the slow time dependence encoded in both the tidal matrix elements themselves and in the orbital elements of $m$, collectively denoted as $\{\Psi\}$, due to possible non-Keplerian local effects taking place in the non-spherically symmetric field of $M$.

Let us, now, assume that the characteristic timescales $P_{\Psi}$ of all the non-Keplerian orbital effects within $S$ are much longer than the orbital period $P^{\prime}{ }_{\mathrm{b}}$ of $S$ itself about $M^{\prime}$. It is a reasonable tenet, satisfied in several astronomical scenarios of potential experimental interest. Thus, it is possible to perform a further average of Equations (25-29) over $P^{\prime}{ }_{\mathrm{b}}$ by keeping $a, e, I, \Omega, \omega$ constant over the integration with respect to some fast variable ${ }^{2}$ of the motion of $M$ around $M^{\prime}$.

The direct effects of order $\mathcal{O}\left(c^{-2}\right)$ can be obtained by evaluating the post-Newtonian tidal matrices Equations $(5,6)$ onto an unchanging Keplerian ellipse as reference unperturbed trajectory.

Below, the averaged tidal matrix elements of Equations (4-6), computed to order $\mathcal{O}\left(c^{-2}\right)$ and to zero order in $J_{2}{ }^{\prime}$, are listed. They are to be inserted in Equations (25-29) to have the direct long-term rates of change of $m$ averaged over $P^{\prime}{ }_{\mathrm{b}}$.

As far as the Newtonian tidal matrix of Equation (4) is concerned, its average, to the zero order in $J_{2}{ }^{\prime}$, is

$$
\begin{aligned}
& \left\langle\mathrm{K}_{11}^{(\mathrm{N})}\right\rangle_{P_{\mathrm{b}}^{\prime}}=-\frac{G M^{\prime}\left(1+3 \cos 2 I^{\prime}+6 \sin ^{2} I^{\prime} \cos 2 \Omega^{\prime}\right)}{8 a^{\prime 3}\left(1-e^{\prime 2}\right)^{3 / 2}} \\
& \left\langle\mathrm{~K}_{22}^{(\mathrm{N})}\right\rangle_{P_{\mathrm{b}}^{\prime}}=-\frac{G M^{\prime}\left(1+3 \cos 2 I^{\prime}-6 \sin ^{2} I^{\prime} \cos 2 \Omega^{\prime}\right)}{8 a^{\prime 3}\left(1-e^{\prime 2}\right)^{3 / 2}} \\
& \left\langle\mathrm{~K}_{33}^{(\mathrm{N})}\right\rangle_{P_{\mathrm{b}}^{\prime}}=\frac{G M^{\prime}\left(1+3 \cos 2 I^{\prime}\right)}{4{a^{\prime}}^{3}\left(1-e^{\prime 2}\right)^{3 / 2}}
\end{aligned}
$$

\footnotetext{
${ }^{2}$ It turned out computationally more convenient to adopt the true anomaly $f^{\prime}$.
} 


$$
\begin{aligned}
\left\langle\mathrm{K}_{12}^{(\mathrm{N})}\right\rangle_{P_{\mathrm{b}}^{\prime}} & =-\frac{3 G M^{\prime} \sin ^{2} I^{\prime} \sin 2 \Omega^{\prime}}{4{a^{\prime}}^{3}\left(1-e^{\prime 2}\right)^{3 / 2},} \\
\left\langle\mathrm{~K}_{13}^{(\mathrm{N})}\right\rangle_{P_{\mathrm{b}}^{\prime}} & =\frac{3 G M^{\prime} \sin 2 I^{\prime} \sin \Omega^{\prime}}{4{a^{\prime}}^{3}\left(1-e^{\prime 2}\right)^{3 / 2}}, \\
\left\langle\mathrm{~K}_{23}^{(\mathrm{N})}\right\rangle_{P_{\mathrm{b}}^{\prime}} & =-\frac{3 G M^{\prime} \sin 2 I^{\prime} \cos \Omega^{\prime}}{4 a^{\prime 3}\left(1-e^{\prime 2}\right)^{3 / 2}} .
\end{aligned}
$$

For the post-Newtonian gravitoelectric tidal field of $M^{\prime}$, Equation (5) yields

$$
\begin{aligned}
& \left\langle\mathrm{K}_{11}^{(\mathrm{GE})}\right\rangle_{P_{\mathrm{b}}}=-\frac{3 G^{2} M^{\prime 2} e^{\prime 2}}{32 c^{2} a^{4}\left(1-e^{\prime 2}\right)^{5 / 2}} \\
& \cdot\left\{12 \cos 2 I^{\prime}+2 \cos 2 \omega^{\prime}-2 \cos 2 I^{\prime} \cos 2 \omega^{\prime}\right. \\
& +\cos 2 \Omega^{\prime}\left[24 \sin ^{2} I^{\prime}+2\left(\cos 2 I^{\prime}+3\right) \cos 2 \omega^{\prime}\right] \\
& \left.-8 \cos I^{\prime} \sin 2 \Omega^{\prime} \sin 2 \omega^{\prime}+4\right\} \text {, } \\
& \left\langle\mathrm{K}_{22}^{(\mathrm{GE})}\right\rangle_{P_{\mathrm{b}}^{\prime}}=\frac{3 G^{2} M^{\prime 2} e^{\prime 2}}{32 c^{2} a^{\prime 4}\left(1-e^{\prime 2}\right)^{5 / 2}} \\
& \cdot\left\{-12 \cos 2 I^{\prime}-2 \cos 2 \omega^{\prime}+2 \cos 2 I^{\prime} \cos 2 \omega^{\prime}\right. \\
& +\cos 2 \Omega^{\prime}\left[24 \sin ^{2} I^{\prime}+2\left(\cos 2 I^{\prime}+3\right) \cos 2 \omega^{\prime}\right] \\
& \left.-8 \cos I^{\prime} \sin 2 \Omega^{\prime} \sin 2 \omega^{\prime}-4\right\}, \\
& \left\langle\mathrm{K}_{33}^{(\mathrm{GE})}\right\rangle_{P_{\mathrm{b}}^{\prime}}=\frac{3 G^{2} M^{\prime 2} e^{\prime 2}}{4 c^{2} a^{\prime 4}\left(1-e^{\prime 2}\right)^{5 / 2}}\left(\sin ^{2} I^{\prime} \cos 2 \omega^{\prime}+3 \cos 2 I^{\prime}+1\right) \text {, } \\
& \left\langle\mathrm{K}_{12}^{(\mathrm{GE})}\right\rangle_{P^{\prime} \mathrm{b}}=-\frac{3 G^{2} M^{\prime 2} e^{\prime 2}}{16 c^{2} a^{\prime 4}\left(1-e^{\prime 2}\right)^{5 / 2}}\left\{4 \cos I^{\prime} \cos 2 \Omega^{\prime} \sin 2 \omega^{\prime}\right. \\
& \left.+\left[12 \sin ^{2} I^{\prime}+\left(\cos 2 I^{\prime}+3\right) \cos 2 \omega^{\prime}\right] \sin 2 \Omega^{\prime}\right\}, \\
& \left\langle\mathrm{K}_{13}^{(\mathrm{GE})}\right\rangle_{P_{\mathrm{b}}^{\prime}}=-\frac{3 G^{2} M^{\prime 2} e^{\prime 2}}{4 c^{2} a^{\prime 4}\left(1-e^{\prime 2}\right)^{5 / 2}} \\
& \text { - }\left\{\sin I^{\prime}\left[\cos \Omega^{\prime} \sin 2 \omega^{\prime}+\cos I^{\prime}\left(\cos 2 \omega^{\prime}-6\right) \sin \Omega^{\prime}\right]\right\}, \\
& \left\langle\mathrm{K}_{23}^{(\mathrm{GE})}\right\rangle_{P^{\prime} \mathrm{b}}=\frac{3 G^{2} M^{\prime 2} e^{\prime 2}}{4 c^{2} a^{\prime 4}\left(1-e^{\prime 2}\right)^{5 / 2}} \\
& \text {. }\left\{\sin I^{\prime}\left[\cos I^{\prime}\left(\cos 2 \omega^{\prime}-6\right) \cos \Omega^{\prime}-\sin 2 \omega^{\prime} \sin \Omega^{\prime}\right]\right\} \text {. }
\end{aligned}
$$

In the limit of $e^{\prime} \rightarrow 0$, Equations (36-41) vanish.

For the post-Newtonian gravitomagnetic tidal field of $M^{\prime}$ due to $J^{\prime}$, from Equation (6) one obtains

$$
\begin{aligned}
& \left\langle\mathrm{K}_{11}^{(\mathrm{GM})}\right\rangle_{P_{\mathrm{b}}^{\prime}}=-\frac{3 G J^{\prime} n_{\mathrm{b}}{ }^{\prime}}{64 c^{2} a^{\prime 3}\left(1-e^{\prime 2}\right)^{3}} \\
& \cdot\left\{40 e^{\prime 2} \sin 2 \omega^{\prime}\left(2 \hat{k}_{z}^{\prime} \cos 2 I^{\prime}+3 \hat{k}_{x}^{\prime} \sin 2 I^{\prime} \sin \Omega^{\prime}\right) \sin 2 \Omega^{\prime}\right. \\
& +5 \cos 2 \omega^{\prime}\left(12 \sin 3 I^{\prime}\left(\hat{k}_{x}{ }^{\prime} \sin \Omega^{\prime}-\hat{k}_{y}^{\prime} \cos \Omega^{\prime}\right) \sin ^{2} \Omega^{\prime}\right.
\end{aligned}
$$

$+\sin I^{\prime}\left(\hat{k}_{y}{ }^{\prime}\left(\cos \Omega^{\prime}+15 \cos 3 \Omega^{\prime}\right)\right.$

$\left.\left.-3 \hat{k}_{x}{ }^{\prime}\left(\sin \Omega^{\prime}+5 \sin 3 \Omega^{\prime}\right)\right)\right) e^{\prime 2}$

$-20\left(3 e^{\prime 2}+2\right) \hat{k}_{z}{ }^{\prime} \cos 3 I^{\prime}-4 \cos I^{\prime}\left(5 \hat{k}_{z}{ }^{\prime} \cos 2 \omega^{\prime}\left(6 \sin ^{2} I^{\prime}\right.\right.$

$\left.+\left(3 \cos 2 I^{\prime}+1\right) \cos 2 \Omega^{\prime}\right) e^{\prime 2}-10 \hat{k}_{y}{ }^{\prime} \sin I^{\prime} \sin 2 \omega^{\prime}$

$\cdot\left(\sin \Omega^{\prime}-3 \sin 3 \Omega^{\prime}\right) e^{\prime 2}+\left(3 e^{\prime 2}+2\right) \hat{k}_{z}{ }^{\prime}$

$\left.\cdot\left(20 \cos 2 \Omega^{\prime} \sin ^{2} I^{\prime}+3\right)\right)$

$$
\begin{aligned}
& +2\left(3 e^{\prime 2}+2\right)\left(20 \sin 3 I^{\prime}\left(\hat{k}_{y}{ }^{\prime} \cos \Omega^{\prime}-\hat{k}_{x}{ }^{\prime} \sin \Omega^{\prime}\right) \sin ^{2} \Omega^{\prime}\right. \\
& \left.\left.+\sin I^{\prime}\left(\hat{k}_{y}{ }^{\prime}\left(\cos \Omega^{\prime}+15 \cos 3 \Omega^{\prime}\right)-3 \hat{k}_{x}{ }^{\prime}\left(\sin \Omega^{\prime}+5 \sin 3 \Omega^{\prime}\right)\right)\right)\right\},
\end{aligned}
$$

$$
\left\langle\mathrm{K}_{22}^{(\mathrm{GM})}\right\rangle_{P^{\prime} \mathrm{b}}=-\frac{3 G J^{\prime} n_{\mathrm{b}}{ }^{\prime}}{64 c^{2}{a^{\prime}}^{3}\left(1-e^{\prime 2}\right)^{3}}
$$

$\cdot\left\{20 e^{\prime 2} \hat{k}_{x}^{\prime}\left(\cos \Omega^{\prime}+3 \cos 3 \Omega^{\prime}\right) \sin 2 I^{\prime} \sin 2 \omega^{\prime}\right.$

$-80 e^{\prime 2} \hat{k}_{z}{ }^{\prime} \cos 2 I^{\prime} \sin 2 \omega^{\prime} \sin 2 \Omega^{\prime}-20\left(3 e^{\prime 2}+2\right) \hat{k}_{z}{ }^{\prime} \cos 3 I^{\prime}$

$+20\left(3 e^{\prime 2} \cos 2 \omega^{\prime}-6 e^{\prime 2}-4\right) \cos ^{2} \Omega^{\prime} \sin 3 I^{\prime}$

$$
\text { · }\left(\hat{k}_{x}{ }^{\prime} \sin \Omega^{\prime}-\hat{k}_{y}{ }^{\prime} \cos \Omega^{\prime}\right)
$$

$+4 e^{\prime 2} \cos I^{\prime}\left(5 \hat{k}_{z}{ }^{\prime} \cos 2 \omega^{\prime}\left(\left(3 \cos 2 I^{\prime}+1\right) \cos 2 \Omega^{\prime}-6 \sin ^{2} I^{\prime}\right)\right.$

$+120 e^{\prime 2} \hat{k}_{y}{ }^{\prime} \cos ^{2} \Omega^{\prime} \sin I^{\prime} \sin 2 \omega^{\prime} \sin \Omega^{\prime}$

$\left.+\left(3 e^{\prime 2}+2\right) \hat{k}_{z}{ }^{\prime}\left(20 \cos 2 \Omega^{\prime} \sin ^{2} I^{\prime}-3\right)\right)$

$+\left(5 e^{\prime 2} \cos 2 \omega^{\prime}+6 e^{\prime 2}+4\right) \sin I^{\prime}\left(3 \hat{k}_{y}{ }^{\prime} \cos \Omega^{\prime}\right.$

$\left.\left.-15 \hat{k}_{y}^{\prime} \cos 3 \Omega^{\prime}-\hat{k}_{x}{ }^{\prime} \sin \Omega^{\prime}+15 \hat{k}_{x}^{\prime} \sin 3 \Omega^{\prime}\right)\right\}$,

$\left\langle\mathrm{K}_{33}^{(\mathrm{GM})}\right\rangle_{P_{\mathrm{b}}^{\prime}}=-\frac{3 G J^{\prime} n_{\mathrm{b}}{ }^{\prime}}{16 c^{2} a^{\prime 3}\left(1-e^{\prime 2}\right)^{3}}$

$\cdot\left\{-20 e^{\prime 2} \sin 2 I^{\prime} \sin 2 \Omega^{\prime}\left(\hat{k}_{x}{ }^{\prime} \cos \Omega^{\prime}+\hat{k}_{y}{ }^{\prime} \sin \Omega^{\prime}\right)\right.$

$+5 e^{\prime 2} \cos 2 \Omega^{\prime}\left(12 \hat{k}_{z}{ }^{\prime} \cos I^{\prime} \sin ^{2} I^{\prime}+\hat{k}_{y}{ }^{\prime} \cos \Omega^{\prime}\left(3 \sin 3 I^{\prime}-\sin I^{\prime}\right)\right.$

$\left.+\hat{k}_{x}^{\prime}\left(\sin I^{\prime}-3 \sin 3 I^{\prime}\right) \sin \Omega^{\prime}\right)$

$+2\left(3 e^{\prime 2}+2\right)\left(3 \hat{k}_{z}{ }^{\prime} \cos I^{\prime}+5 \hat{k}_{z}{ }^{\prime} \cos 3 I^{\prime}\right.$

$\left.\left.-\left(\sin I^{\prime}+5 \sin 3 I^{\prime}\right)\left(\hat{k}_{y}{ }^{\prime} \cos \Omega^{\prime}-\hat{k}_{x}{ }^{\prime} \sin \Omega^{\prime}\right)\right)\right\}$,

$\left\langle\mathrm{K}_{12}^{(\mathrm{GM})}\right\rangle_{P^{\prime} \mathrm{b}}=-\frac{3 G J^{\prime} n_{\mathrm{b}}{ }^{\prime}}{64 c^{2} a^{\prime 3}\left(1-e^{\prime 2}\right)^{3}}$

$\cdot\left\{-80 e^{\prime 2} \hat{k}_{z}{ }^{\prime} \cos 2 I^{\prime} \cos 2 \Omega^{\prime} \sin 2 \omega^{\prime}\right.$

$+20 e^{\prime 2} \sin 2 I^{\prime} \sin 2 \omega^{\prime}\left(\hat{k}_{y}{ }^{\prime} \cos \Omega^{\prime}+3 \hat{k}_{y}{ }^{\prime} \cos 3 \Omega^{\prime}\right.$

$\left.+\hat{k}_{x}^{\prime}\left(\sin \Omega^{\prime}-3 \sin 3 \Omega^{\prime}\right)\right)$ 


$$
\begin{aligned}
& -10 \hat{k}_{z}^{\prime} \cos 3 I^{\prime}\left(3 e^{\prime 2} \cos 2 \omega^{\prime}-6 e^{\prime 2}-4\right) \sin 2 \Omega^{\prime} \\
& -10 \hat{k}_{z}{ }^{\prime} \cos I^{\prime}\left(5 e^{\prime 2} \cos 2 \omega^{\prime}+6 e^{\prime 2}+4\right) \sin 2 \Omega^{\prime} \\
& +10\left(3 e^{\prime 2} \cos 2 \omega^{\prime}-6 e^{\prime 2}-4\right) \sin 3 I^{\prime} \\
& \cdot\left(\hat{k}_{y}{ }^{\prime} \cos \Omega^{\prime}-\hat{k}_{x}{ }^{\prime} \sin \Omega^{\prime}\right) \sin 2 \Omega^{\prime} \\
& +\left(5 e^{\prime 2} \cos 2 \omega^{\prime}+6 e^{\prime 2}+4\right) \sin I^{\prime}\left(\hat{k}_{x}{ }^{\prime} \cos \Omega^{\prime}+15 \hat{k}_{x}{ }^{\prime} \cos 3 \Omega^{\prime}\right. \\
& \left.\left.-\hat{k}_{y}^{\prime} \sin \Omega^{\prime}+15 \hat{k}_{y}^{\prime} \sin 3 \Omega^{\prime}\right)\right\}, \\
& \left\langle\mathrm{K}_{13}^{(\mathrm{GM})}\right\rangle_{P^{\prime} \mathrm{b}}=\frac{3 G J^{\prime} n_{\mathrm{b}}{ }^{\prime}}{32 c^{2}{a^{\prime}}^{3}\left(1-e^{\prime 2}\right)^{3}} \\
& \cdot\left\{-10 e^{\prime 2} \hat{k}_{z}^{\prime} \cos 2 \omega^{\prime}\left(\sin I^{\prime}-3 \sin 3 I^{\prime}\right) \sin \Omega^{\prime}\right. \\
& +40 e^{\prime 2} \sin 2 \omega^{\prime}\left(\hat{k}_{z}^{\prime} \cos \Omega^{\prime} \sin 2 I^{\prime}\right. \\
& \left.+\cos 2 I^{\prime}\left(\hat{k}_{y}^{\prime} \cos 2 \Omega^{\prime}-\hat{k}_{x}^{\prime} \sin 2 \Omega^{\prime}\right)\right) \\
& -4\left(3 e^{\prime 2}+2\right) \hat{k}_{z}^{\prime}\left(\sin I^{\prime}+5 \sin 3 I^{\prime}\right) \sin \Omega^{\prime} \\
& +5 e^{\prime 2} \cos 3 I^{\prime}\left(3 \cos 2 \omega^{\prime}\left(\hat{k}_{x}{ }^{\prime} \cos 2 \Omega^{\prime}+\hat{k}_{y}{ }^{\prime} \sin 2 \Omega^{\prime}\right)\right. \\
& \left.+2\left(3 e^{\prime 2}+2\right) \hat{k}_{x}^{\prime}\right) \\
& +e^{\prime 2} \cos I^{\prime}\left(5 \operatorname { c o s } 2 \omega ^ { \prime } \left(12 \hat{k}_{x}^{\prime} \sin ^{2} I^{\prime}\right.\right. \\
& \left.+5 \hat{k}_{x}^{\prime} \cos 2 \Omega^{\prime}+5 \hat{k}_{y}^{\prime} \sin 2 \Omega^{\prime}\right) \\
& +2\left(3 e^{\prime 2}+2\right)\left(2 0 \left(\hat{k}_{x}{ }^{\prime} \cos 2 \Omega^{\prime}\right.\right. \\
& \left.\left.\left.\left.+\hat{k}_{y}^{\prime} \sin 2 \Omega^{\prime}\right) \sin ^{2} I^{\prime}+3 \hat{k}_{x}^{\prime}\right)\right)\right\}, \\
& \left\langle\mathrm{K}_{23}^{(\mathrm{GM})}\right\rangle_{P_{\mathrm{b}}^{\prime}}=-\frac{3 G J^{\prime} n_{\mathrm{b}}^{\prime}}{64 c^{2}{a^{\prime}}^{3}\left(1-e^{\prime 2}\right)^{3}} \\
& \cdot\left\{1 0 e ^ { \prime 2 } \operatorname { c o s } 2 \omega ^ { \prime } \left(-12 \hat{k}_{y}{ }^{\prime} \cos I^{\prime} \sin ^{2} I^{\prime}\right.\right. \\
& +\hat{k}_{y}{ }^{\prime}\left(5 \cos I^{\prime}+3 \cos 3 I^{\prime}\right) \cos 2 \Omega^{\prime} \\
& -2 \hat{k}_{z}{ }^{\prime} \cos \Omega^{\prime}\left(\sin I^{\prime}-3 \sin 3 I^{\prime}\right) \\
& \left.-\hat{k}_{x}^{\prime}\left(5 \cos I^{\prime}+3 \cos 3 I^{\prime}\right) \sin 2 \Omega^{\prime}\right) \\
& -80 e^{\prime 2} \sin 2 \omega^{\prime}\left(\hat{k}_{z}{ }^{\prime} \sin 2 I^{\prime} \sin \Omega^{\prime}\right. \\
& \left.+\cos 2 I^{\prime}\left(\hat{k}_{x}{ }^{\prime} \cos 2 \Omega^{\prime}+\hat{k}_{y}{ }^{\prime} \sin 2 \Omega^{\prime}\right)\right) \\
& +4\left(3 e^{\prime 2}+2\right)\left(-5 \hat{k}_{y}^{\prime} \cos 3 I^{\prime}\right. \\
& -2 \hat{k}_{z}{ }^{\prime} \cos \Omega^{\prime}\left(\sin I^{\prime}+5 \sin 3 I^{\prime}\right) \\
& \left.\left.+\cos I^{\prime}\left(20 \sin ^{2} I^{\prime}\left(\hat{k}_{y}{ }^{\prime} \cos 2 \Omega^{\prime}-\hat{k}_{x}{ }^{\prime} \sin 2 \Omega^{\prime}\right)-3 \hat{k}_{y}{ }^{\prime}\right)\right)\right\} \text {. }
\end{aligned}
$$

Note that Equations (42-47) have a general validity since they are restricted neither to any specific spatial orientation of the spin axis of $M^{\prime}$ nor to circular and/or equatorial orbits of $M$ about $M^{\prime}$.

It should be remarked that indirect, mixed effects of order $\mathcal{O}\left(c^{-2}\right)$ arise, in principle, also from the Newtonian tidal matrix of Equation (4) when the post-Newtonian effects of the field of $M^{\prime}$ onto the orbital motion of $M$ are taken into account (Mashhoon et al., 1989). The same holds also with $J_{2}{ }^{\prime}$, accounting for possible deviations of $M^{\prime}$ from spherical symmetry at the Newtonian level itself. The calculation of such further effects, whose size may be comparable with that of the direct ones, is beyond the scope of this paper.

\section{POSSIBLE SCENARIOS OF INTEREST FOR EMPIRICAL TESTS}

Forthcoming space-based missions to astronomical bodies orbiting large primaries such as the Sun and Jupiter, in conjunction with expected progresses in interplanetary tracking techniques (Iess and Asmar, 2007; Iess et al., 2009, 2014), may, in principle, represent an opportunity to put on the test the post-Newtonian tidal effects calculated in the previous sections.

Let us consider the forthcoming BepiColombo ${ }^{3}$ (Benkhoff et al., 2010) and JUICE ${ }^{4}$ (Grasset et al., 2013) missions targeted to Mercury and the Jovian natural satellite Ganymede, respectively; in the following, the symbol $\iota$ will be adopted for the inclinations of the orbital planes of such spacecrafts to the equators of the orbited bodies. A probe named Mercury Planetary Orbiter $^{5}$ (MPO) (Ashby et al., 2007) is planned to be released in a polar $\left(\iota_{\mathrm{MPO}}=90^{\circ}\right)$, elliptical orbit $\left(e_{\mathrm{MPO}}=0.16\right)$ around Mercury with an orbital period of approximately $2.3 \mathrm{~h}\left(a_{\mathrm{MPO}}=\right.$ $3,394 \mathrm{~km}$ ). The nominal science duration is one year, with a possible extension of another year. Importantly, orbital maneuvers to change the attitude of the spacecraft are scheduled every about $44 \mathrm{~d}$, so that long smooth orbital arcs should be available. The JUICE mission (Grasset et al., 2013) to the Jovian system will culminate in a dedicated orbital tour around Ganymede which should encompass a $30 \mathrm{~d}$ science phase during which JUICE will orbit $^{6}$ the satellite in a polar, circular low path with an altitude as little as $h=200 \mathrm{~km}$. Table 1 summarizes the characteristic frequencies of both the scenarios considered, showing that our results of the previous sections are applicable to them. In Table 2, we maximize the values of the post-Newtonian tidal perturbations for both MPO and JGO with respect to their unknown node $\Omega$ and pericenter $\omega$. We do the same also for some of the most important competing Newtonian and post-Newtonian orbital perturbations. It can be noticed that, while for MPO the gravitoelectric tidal effects are larger than the gravitomagnetic ones, the situation is reversed for JGO because of its scheduled zero eccentricity. As far as the magnitudes of the tidal effects are concerned, they are larger for JGO; its gravitomagnetic tidal precessions reach the $\approx 10^{-1}-10^{-2}$ mas $\mathrm{yr}^{-1}$ level. In principle, a

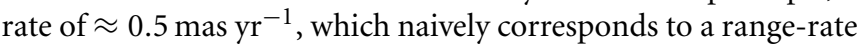
as little as $\approx 0.05 \mathrm{~mm} \mathrm{~s}^{-1}$ at the distance of Jupiter from us, might be detectable with the expected improvement down to $0.01 \mathrm{~mm}$ $\mathrm{s}^{-1}$ at $60 \mathrm{~s}$ integration time in the Doppler range-rate techniques from the ASTRA study (Iess et al., 2014). Unfortunately, such figures are too small if compared with those of the competing

\footnotetext{
${ }^{3}$ See also http://sci.esa.int/bepicolombo/ on the Internet.

${ }^{4}$ See also http://sci.esa.int/juice/ on the Internet.

${ }^{5}$ See http://sci.esa.int/bepicolombo/48872-spacecraft/ on the Internet.

${ }^{6}$ See http://sci.esa.int/juice/50074-scenario-operations/ on the Internet.
} 
Table 1 | Some characteristic orbital frequencies, in $\mathrm{s}^{-1}$, of the Sun-Mercury-Mercury Planetary Orbiter (MPO) and of the Jupiter-Ganymede-Jupiter Ganymede Orbiter (JGO) (Grasset et al., 2013) systems.

Frequency

(Sun-Mercury-MPO)

\begin{tabular}{|c|c|c|c|}
\hline$\dot{\omega}_{\zeta}^{(\mathrm{GM})}$ & $1 \times 10^{-18}$ & $\dot{\Omega}_{\mathrm{Gan}}^{(\mathrm{GM})}$ & $8 \times 10^{-16}$ \\
\hline$\dot{\omega}_{\breve{\dagger}}^{\left(J_{2}^{\odot}\right)}$ & $8 \times 10^{-17}$ & $\dot{\varpi}_{G a n}^{\left(J_{2}^{4}\right)}$ & $3 \times 10^{-9}$ \\
\hline$\dot{\omega}_{\breve{\zeta}}^{(\mathrm{GE})}$ & $7 \times 10^{-14}$ & $\dot{\omega}_{\mathrm{Gan}}^{(\mathrm{GE})}$ & $4 \times 10^{-14}$ \\
\hline$n_{\varnothing}$ & $8 \times 10^{-7}$ & $n_{\text {Gan }}$ & $1 \times 10^{-5}$ \\
\hline$\dot{\Omega}_{\mathrm{MPO}}^{(\mathrm{GM})}$ & $3 \times 10^{-17}$ & $\dot{\Omega}_{\mathrm{JGO}}^{(\mathrm{GM})}$ & $2 \times 10^{-16}$ \\
\hline$\dot{\omega}_{\mathrm{MPO}}^{(\mathrm{GE})}$ & $2 \times 10^{-13}$ & $\dot{\omega}_{\mathrm{JGO}}^{(\mathrm{GE})}$ & $8 \times 10^{-14}$ \\
\hline$\dot{\omega}_{\mathrm{MPO}}^{\left(\begin{array}{c}\forall \\
J_{2}^{+}\end{array}\right)}$ & $6 \times 10^{-9}$ & $\left|\dot{\omega}_{\mathrm{JGO}}^{\left(J_{2}^{\mathrm{Gan}}\right)}\right|$ & $5 \times 10^{-8}$ \\
\hline$n_{\mathrm{MPO}}$ & $7 \times 10^{-4}$ & $n_{\mathrm{JGO}}$ & $6 \times 10^{-4}$ \\
\hline
\end{tabular}

For JGO, we considered the planned 30 days phase of the Ganymede tour to be spent in a low altitude $(200 \mathrm{~km})$ circular orbit (http://sci.esa.int/juice/ 50074-scenario-operations/). For the sake of simplicity, the Newtonian and the post-Newtonian precessions due to the oblateness $\mathrm{J}_{2}$ and to the angular momentum $\mathrm{J}$ of the primaries were calculated in equatorial coordinate systems. Indeed, while both MPO and JGO will move along polar trajectories at $\iota=90^{\circ}$ to the equators of their primaries, the orbits of Mercury and Ganymede lie almost in the equatorial planes of the Sun $\left(\iota_{\gamma}=3.38^{\circ}\right)$ and of Jupiter $\left(\iota_{\mathrm{Gan}}=\right.$ $0.20^{\circ}$ ), respectively. For Ganymede, the value $\mathrm{J}_{2}^{\text {Gan }}=1.27 \times 10^{-4}$ (Anderson et al., 1996) was adopted, while its angular momentum JGan $=3 \times 10^{30} \mathrm{~kg}$ $\mathrm{m}^{2} \mathrm{~s}^{-1}$ was inferred from the values of its mass, equatorial radius and normalized polar moment of inertia (Anderson et al., 1996). For Mercury, we assumed $J_{2}^{\not}=1.92 \times 10^{-5}$ (Smith et al., 2010), while its angular momentum $J^{\not}=8.4 \times 10^{29} \mathrm{~kg} \mathrm{~m}^{2} \mathrm{~s}^{-1}$ was obtained from the latest determinations of its equatorial radius (Byrne et al., 2014) and normalized polar moment of inertia (Margot et al., 2012). For the angular momentum and the oblateness of the Sun and of Jupiter, we assumed $J^{\odot}=1.90 \times 10^{41} \mathrm{~kg} \mathrm{~m}^{2} \mathrm{~s}^{-1}$ (Pijpers, 1998), $J_{2}^{\odot}=2.1 \times 10^{-7}$ (Folkner et al., 2014), and $J^{4}=6.9 \times 10^{38} \mathrm{~kg} \mathrm{~m}^{2} \mathrm{~s}^{-1}$ (Soffel et al., 2003), $J_{2}^{4}=1.469 \times 10^{-2}$ (Jacobson, 2003), respectively.

orbital effects. Suffice it to say that the oblateness of Ganymede is presently known with a relative uncertainty of the order of just $\sigma_{J_{2}} / J_{2}=2 \times 10^{-2}$ (Anderson et al., 1996). An improvement of $6-7$ orders of magnitude is beyond the goals of the JUICE mission itself (Grasset et al., 2013). Strictly speaking, such considerations hold only for the direct post-Newtonian tidal effects calculated in the previous sections; the total sensitivity budget should account for the indirect, mixed effects of order $\mathcal{O}\left(c^{-2}\right)$ as well.

In principle, a rather unconventional possibility could be the realization of an artificial mini-planetary system to be carried onboard a drag-free spacecraft orbiting, say, the Earth; such an idea was already proposed in the past to accurately measure the Newtonian constant of gravitation $G$ (Nobili et al., 1987, 1988, 1990; Keyser, 1992; Sanders and Deeds, 1992; Sanders et al., 1999, 2000), and more recently, to put on the test the MOND
Table 2 | Maximum nominal values of the direct orbital rates of change $\dot{\Psi}$ of MPO and JGO, averaged over both $P_{\mathrm{b}}$ and $\boldsymbol{P}_{\mathrm{b}}^{\prime}$, induced by the post-Newtonian gravitoelectric and gravitomagnetic tidal field of $M^{\prime}$, by its Newtonian tidal field, and by some competing Newtonian and post-Newtonian perturbations due to the deviation from spherical symmetry of the field of $M$.

\begin{tabular}{|c|c|c|c|}
\hline$\dot{\Psi}$ (MPO) & Value & $\dot{\Psi}$ (JGO) & Value \\
\hline$\dot{e}^{(\text {tid GM) }}$ & $1 \times 10^{-21} \mathrm{~s}^{-1}$ & $\dot{e}^{(\text {tid GM) }}$ & $0 s^{-1}$ \\
\hline$j^{\prime}$ (tid GM) & $4 \times 10^{-6}$ mas $_{y^{-1}}$ & j(tid GM) & 0.01 mas $\mathrm{yr}^{-1}$ \\
\hline$\dot{\Omega}^{\text {(tid GM) }}$ & $1 \times 10^{-5}$ mas $_{y^{-1}}$ & $\dot{\Omega}^{(\mathrm{tid} G M)}$ & 0.07 mas $\mathrm{yr}^{-1}$ \\
\hline$\dot{\omega}^{(\text {tid GM) }}$ & $8 \times 10^{-5}$ mas $r^{-1}$ & $\dot{\omega}^{(\mathrm{tid} G M)}$ & 0.54 mas $\mathrm{yr}^{-1}$ \\
\hline$\dot{e}^{(\text {tid GE) }}$ & $1 \times 10^{-18} \mathrm{~s}^{-1}$ & $\dot{e}^{(\text {tid GE) }}$ & $0 s^{-1}$ \\
\hline j(tid GE) & $0.0025 \mathrm{mas} \mathrm{yr}^{-1}$ & $j^{(\text {tid GE) }}$ & $8 \times 10^{-7} \mathrm{mas} \mathrm{yr}^{-1}$ \\
\hline$\dot{\Omega}^{(\text {tid GE) }}$ & $0.0084{\text { mas } \mathrm{yr}^{-1}}^{-1}$ & $\dot{\Omega}^{(\mathrm{tid} G E)}$ & $2 \times 10^{-6}{\mathrm{mas} \mathrm{yr}^{-1}}^{-1}$ \\
\hline$\dot{\omega}^{(\mathrm{tid} \mathrm{GE})}$ & $0.0458 \mathrm{mas} \mathrm{yr}^{-1}$ & $\dot{\omega}^{(\text {tid GE) }}$ & $1 \times 10^{-5}{\text { mas } y r^{-1}}^{-1}$ \\
\hline j(GM) & 0.1038 mas $r^{-1}$ & $j(\mathrm{GM})$ & 0.59 mas $\mathrm{yr}^{-1}$ \\
\hline$\dot{\Omega}^{(\mathrm{GM})}$ & $0.1907 \mathrm{mas} \mathrm{yr}^{-1}$ & $\dot{\Omega}^{(\mathrm{GM})}$ & $1.24 \mathrm{mas} \mathrm{yr}^{-1}$ \\
\hline$\dot{\omega}^{(\mathrm{GM})}$ & 0.2075 mas $r^{-1}$ & $\dot{\omega}^{(\mathrm{GM})}$ & $1.18{\text { mas } \mathrm{yr}^{-1}}^{-1}$ \\
\hline$\dot{\omega}^{(\mathrm{GE})}$ & $1087.78{\text { mas } \mathrm{yr}^{-1}}^{-1}$ & $\dot{\omega}^{(\mathrm{GE})}$ & 499.6 mas $r^{-1}$ \\
\hline$\dot{e}^{(\mathrm{tid} N)}$ & $2.9 \times 10^{-10} \mathrm{~s}^{-1}$ & $\dot{e}^{(t i d N)}$ & $0 s^{-1}$ \\
\hline$\dot{j}($ tid $\mathrm{N})$ & $6.7 \times 10^{5} \mathrm{mas} \mathrm{yr}^{-1}$ & $j($ tid N $)$ & $7 \times 10^{7}$ mas yr $^{-1}$ \\
\hline$\dot{\Omega}^{(\text {tid N })}$ & $2 \times 10^{6}$ mas yr $^{-1}$ & $\dot{\Omega}^{(\text {tid N) }}$ & $3 \times 10^{8}{\text { mas } y r^{-1}}^{-1}$ \\
\hline$\dot{\omega}^{(\text {tid N })}$ & $1.3 \times 10^{7} \mathrm{mas} \mathrm{yr}^{-1}$ & $\dot{\omega}^{(\text {tid N)}}$ & $2 \times 10^{9}$ mas yr $^{-1}$ \\
\hline$j\left(J_{2}\right)$ & $8 \times 10^{6}$ mas yr $^{-1}$ & $j\left(J_{2}\right)$ & $6 \times 10^{7}$ mas yr $^{-1}$ \\
\hline$\dot{\Omega}^{\left(J_{2}\right)}$ & $3 \times 10^{7}$ mas yr $^{-1}$ & $\dot{\Omega}^{\left(J_{2}\right)}$ & $2.7 \times 10^{8} \mathrm{mas} \mathrm{yr}^{-1}$ \\
\hline$\dot{\omega}^{\left(J_{2}\right)}$ & $4 \times 10^{7}$ mas yr $^{-1}$ & $\dot{\omega}^{\left(J_{2}\right)}$ & $3.5 \times 10^{8} \mathrm{mas} \mathrm{yr}^{-1}$ \\
\hline
\end{tabular}

The values of $\Omega_{\max }$ and $\omega_{\max }$, which are different for each orbital effect considered, are not reported. The units for the precessions are milliarcseconds per year (mas $\mathrm{yr}^{-1}$ ), apart from the eccentricity e whose rate of change is expressed in $\mathrm{s}^{-1}$. The Newtonian $J_{2}$ and the post-Newtonian $\mathrm{J}$ orbital precessions for a generic orientation of the spin axis of $M$ were retrieved from lorio (2011). The mean equinox and the mean equatorial plane of the Earth at the epoch J2000.0 of the International Celestial Reference Frame (ICRF) were adopted for both $\mathcal{K}$ and $\mathcal{K}^{\prime}$. The orientations of the spin axes with respect to the ICRF were retrieved from Seidelmann et al. (2007).

theory (Sahni and Shtanov, 2008). The conditions of validity of the present analysis could be fulfilled, e.g., by placing a nonrotating sphere made of tungsten with density $\rho_{\mathrm{W}}=19.6 \mathrm{~g} \mathrm{~cm}^{-3}$ and $M=28 \mathrm{~kg}, R=7 \mathrm{~cm}$ inside a drag-free spacecraft orbiting the Earth in some suitably chosen High Earth Orbit (HEO). By assuming, say, $a=10 \mathrm{~cm}$ for the test particle orbiting the tungsten sphere and a geostationary orbit with $a^{\prime}=42,164 \mathrm{~km}$ for the spacecraft, it would be possible to obtain

$$
\frac{n_{\mathrm{b}}^{\prime}}{n_{\mathrm{b}}}=5 \times 10^{-2} .
$$

Moreover, the local dynamics of such a spaceborne artificial planetary system would be practically free from systematic nonKeplerian gravitational perturbations due to $M$. Indeed, careful manufacturing of the sphere would allow to make its oblateness negligible; the post-Newtonian gravitoelectric pericenter precession would be completely irrelevant being as little as $\dot{\omega} \approx$ $10^{-12}$ mas $\mathrm{yr}^{-1}$. Table 3 summarizes the nominal maximum 
Table 3 | Maximum nominal values of the direct orbital rates of change $\dot{\Psi}$ (here, $\varpi \doteq \Omega+\omega$ is the longitude of the pericenter) of a member of a spaceborne artificial mini-planetary system orbiting a tungsten sphere of mass $M=28 \mathrm{~kg}$ and radius $R=7 \mathrm{~cm}$ in a circular orbit with $a=10 \mathrm{~cm}, e=0, I=90^{\circ}$ averaged over both $P_{\mathrm{b}}$ and $P_{b}^{\prime}$, induced by the post-Newtonian gravitoelectric and gravitomagnetic tidal field of the Earth and by its Newtonian tidal field.

\begin{tabular}{|c|c|}
\hline$\dot{\Psi}$ & Value \\
\hline$\dot{e}^{(\mathrm{tid} G M)}$ & $0 s^{-1}$ \\
\hline j(tid GM) & 0 mas $\mathrm{yr}^{-1}$ \\
\hline$\dot{\varpi}^{\text {(tid GM) }}$ & 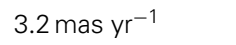 \\
\hline$\dot{e}^{(\text {tid GE) }}$ & $0 s^{-1}$ \\
\hline$\dot{j}^{(\text {tid GE })}$ & $32.8 \mathrm{mas} \mathrm{yr}^{-1}$ \\
\hline$\dot{\varpi}^{\text {(tid GE) }}$ & 189.6 mas $r^{-1}$ \\
\hline$\dot{e}^{(\mathrm{tid} N)}$ & $0 s^{-1}$ \\
\hline j(tid N) & $2.6 \times 10^{10} \mathrm{mas} \mathrm{yr}^{-1}$ \\
\hline$\dot{\varpi}^{(\text {tid N) }}$ & $1.5 \times 10^{11} \mathrm{mas} \mathrm{yr}^{-1}$ \\
\hline
\end{tabular}

For the spacecraft hosting it we assumed an highly elliptical geostationary polar orbit characterized by $a^{\prime}=42,164 \mathrm{~km}, e^{\prime}=0.7, l^{\prime}=\omega^{\prime}=90^{\circ}, \Omega^{\prime}=0^{\circ}$. The values of $\Omega_{\max }$ and $\omega_{\max }$, which are different for each orbital effect considered, are not reported. The units for the precessions are milliarcseconds per year (mas $\mathrm{yr}^{-1}$ ), apart from the eccentricity e whose rate of change is expressed in $\mathrm{s}^{-1}$. The mean equinox and the mean equatorial plane of the Earth at the epoch J2000.0 of the International Celestial Reference Frame (ICRF) were adopted for both $\mathcal{K}$ and $\mathcal{K}^{\prime}$. The orientation of the Earth's spin axis with respect to the ICRF was retrieved from Seidelmann et al. (2007).

values of the precessions of a "planet" orbiting the aforementioned tungsten sphere along a circular path perpendicular to the Earth's equator in a spacecraft following a highly eccentric polar orbit around the Earth. In fact, the size of the post-Newtonian tidal effects are not negligible. Nonetheless, the tidal precessions of Newtonian origin would overwhelm them since the gravitational parameter $G M^{\prime}$ of the Earth is currently known with a $2 \times 10^{-9}$ relative accuracy (Petit and Luzum, 2010), insufficient by $1-2$ orders of magnitude for our purposes.

\section{OVERVIEW AND CONCLUSIONS}

We looked at the direct long-term orbital rates of change occurring within a local gravitationally bound two-body system as gradiometers to potentially detect post-Newtonian tidal effects due to its slow motion in the external field of a distant third body. We also assumed that the characteristic orbital frequencies of the internal dynamics of the local binary are quite smaller than the frequency of its orbital motion around the external source. We obtained general analytical expressions valid for arbitrary orbital configurations and for a generic orientation of the spin axis of the external body. Future work should be devoted to the calculation of the indirect, mixed post-Newtonian effects arising from the interplay between the Newtonian tidal matrix and the post-Newtonian orbital motion of the binary in the external field.

We applied our results to the future BepiColombo and JUICE man-made missions to Mercury and Ganymede, respectively. It turned out that that, although the expected improvements in interplanetary tracking may, perhaps, allow for a detection of the tidal effects we are interested in, especially for JUICE, the impact of several competing orbital effects of Newtonian and post-Newtonian origin, acting as sources of potential systematic errors, should be carefully considered.

Another possibility which, in principle, may be further pursued is the realization of an artificial mini-planetary system to be carried onboard an Earth-orbiting drag-free spacecraft. If, on the one hand, the post-Newtonian tidal precessions occurring in such a system may be relatively large, amounting to about $1-10^{2}$ mas $\mathrm{yr}^{-1}$, on the other hand, the product of the Earth's mass times the Newtonian gravitational constant is currently known with insufficient accuracy to allow for an effective subtraction of the competing Newtonian tidal precessions.

\section{REFERENCES}

Anderson, J. D., Lau, E. L., Sjogren, W. L., Schubert, G., and Moore, W. B. (1996) Gravitational constraints on the internal structure of Ganymede. Nature 384, 541-543. doi: 10.1038/384541a0

Ashby, N., Bender, P. L., and Wahr, J. M. (2007). Future gravitational physics tests from ranging to the BepiColombo Mercury planetary orbiter. Phys. Rev. D 75:022001. doi: 10.1103/PhysRevD.75.022001

Benkhoff, J., van Casteren, J., Hayakawa, H., Fujimoto, M., Laakso, H., Novara, M., et al. (2010). BepiColombo-Comprehensive exploration of Mercury: mission overview and science goals. Planet. Space Sci. 58, 2-20. doi: 10.1016/j.pss.2009.09.020

Bertotti, B., Farinella, P., and Vokrouhlický, D. (2003). Physics of the Solar System. Dordrecht: Kluwer Academic Press. doi: 10.1007/978-94-010-0233-2

Braginsky, V. B., and Polnarev, A. G. (1980). Relativistic spin-quadrupole gravitational effect. J. Exp. Theor. Phys. Lett. 31, 415-418.

Brumberg, V. A. (1991). Essential Relativistic Celestial Mechanics. Bristol: Adam Hilger.

Brumberg, V. A., and Kopeikin, S. M. (1989). Relativistic reference systems and motion of test bodies in the vicinity of the Earth. Nuovo Cimento B 103, 63-98. doi: $10.1007 / \mathrm{BF} 02888894$

Burns, J. A. (1976). Elementary derivation of the perturbation equations of celestial mechanics. Am. J. Phys. 44, 944-949. doi: 10.1119/1.10237

Byrne, P. K., Klimczak, C., Celâl Șengör, A. M., Solomon, S. C., Watters, T. R., and Hauck, S. A. II. (2014). Mercury's global contraction much greater than earlier estimates. Nat. Geosci. 7, 301-307. doi: 10.1038/ ngeo2097

Chicone, C., and Mashhoon, B. (2002). The generalized Jacobi equation. Class. Quant. Grav. 19, 4231-4248. doi: 10.1088/0264-9381/19/16/301

Chicone, C., and Mashhoon, B. (2006). Tidal dynamics in Kerr spacetime. Class. Quant. Grav. 23, 4021-4033. doi: 10.1088/0264-9381/23/ $12 / 002$

de Sitter, W. (1916). On Einstein's theory of gravitation and its astronomical consequences. Second paper. Monthly notices of the royal astronomical society. 77, 155-184. doi: 10.1093/mnras/77.2.155

Einstein, A. (1915). Erklärung der perihelbewegung des merkur aus der allgemeinen relativitätstheorie. Sitzungsberichte der Preußischen Akademie der Wissenschaften 47, 831-839.

Fermi, E. (1922). Sopra i fenomeni che avvengono in vicinanza di una linea oraria. Rendiconti dei Lincei 31, 21-23; 51-52.

Fokker, A. D. (1921). The geodesic precession: a consequence of Einsteinss theory of graviation. Koninklijke Nederlandsche Akademie van Wetenschappen 23, 729-738.

Folkner, W. M., Williams, J. G., Boggs, D. H., Park, R. S., and Kuchynka, P. (2014). The Planetary and Lunar Ephemerides DE430 and DE431. Interplanet. Netw. Prog. Rep 196:C1.

Gill, E., Schastok, J., Soffel, M. H., and Ruder, H. (1989). On the MashhoonTheiss "anomaly". Phys. Rev. D 39, 2441-2443. doi: 10.1103/PhysRevD. 39.2441

Grasset, O., Dougherty, M. K., Coustenis, A., Bunce, E. J., Erd, C., Titov, D., et al. (2013). JUpiter ICy moons Explorer (JUICE): an ESA mission to orbit Ganymede and to characterise the Jupiter system. Planet. Space Sci. 78, 1-21. doi: 10.1016/j.pss.2012.12.002 
Iess, L., and Asmar, S. (2007). Probing space-time in the solar system:. from cassini to bepicolombo. Int. J. Modern Phys. D 16, 2117-2126. doi: 10.1142/S0218271807011449

Iess, L., Asmar, S., and Tortora, P. (2009). MORE: An advanced tracking experiment for the exploration of Mercury with the mission BepiColombo. Acta Astronautica 65, 666-675. doi: 10.1016/j.actaastro.2009. 01.049

Iess, L., Di Benedetto, M., James, N., Mercolino, M., Simone, L., and Tortora, P. (2014). Astra: interdisciplinary study on enhancement of the end-to-end accuracy for spacecraft tracking techniques. Acta Astronautica 94, 699-707. doi: 10.1016/j.actaastro.2013.06.011

Iorio, L. (2011). Perturbed stellar motions around the rotating black hole in Sgr $\mathrm{A}^{*}$ for a generic orientation of its spin axis. Phys. Rev. D 84:124001. doi: 10.1103/PhysRevD.84.124001

Jacobson, R. A. (2003). JUP230 orbit solution. NASA Tech. Memorandum.

Keyser, P. T. (1992). Perturbative forces on an artificial binary for measuring G. Phys. Lett. A 167, 29-31. doi: 10.1016/0375-9601(92)90621-R

Kopeikin, S., Efroimsky, M., and Kaplan, G. (2011). Relativistic Celestial Mechanics of the Solar System. New York, NY: Wiley. doi: 10.1002/9783527634569

Lense, J., and Thirring, H. (1918). Über den Einfluß der Eigenrotation der Zentralkörper auf die Bewegung der Planeten und Monde nach der Einsteinschen Gravitationstheorie. Physikalische Zeitschrift 19, $156-163$.

Levi-Civita, T. (1926). Sur l' écart géodésique. Matematische Annalen 97, 291-320. doi: 10.1007/BF01447869

Li, X.-Q., Shao, M.-X., Paik, H. J., Huang, Y.-C., Song, T.-X., and Bian, $X$. (2014). Effects of satellite positioning errors and earths multipole moments in the detection of the gravitomagnetic field with an orbiting gravity gradiometer. Gen. Relat. Gravit. 46, 1-14. doi: 10.1007/s10714-0141737-8

Margot, J.-L., Peale, S. J., Solomon, S. C., Hauck, II, S. A., Ghigo, F. D., Jurgens, R. F., et al. (2012). Mercury's moment of inertia from spin and gravity data. J. Geophys. Res. 117. doi: 10.1029/2012JE004161

Mashhoon, B. (1977). Tidal radiation. Astrophys. J. 216, 591-609.

Mashhoon, B., Paik, H. J., and Will, C. M. (1989). Detection of the gravitomagnetic field using an orbiting superconducting gravity gradiometer. Theoretical principles. Phys. Rev. D 39, 2825-2838. doi: 10.1103/PhysRevD. 39.2825

Mashhoon, B., and Theiss, D. S. (1982). Relativistic tidal forces and the possibility of measuring them. Phys. Rev. Lett. 49, 1542-1545. doi: 10.1103/PhysRevLett.49.1542

Mashhoon, B., and Theiss, D. S. (1986). Gravitational influence of the rotation of the sun on the earth-moon system. Phys. Lett. A 115, 333-337. doi: 10.1016/0375-9601(86)90625-0

Mashhoon, B., and Theiss, D. S. (1991). Relativistic lunar theory. Nuovo Cimento B 106, 545-571. doi: 10.1007/BF02726789

Mashhoon, B., and Theiss, D. S. (2001). "Relativistic effects in the motion of the moon," in Gyros, Clocks, Interferometers .... Testing Relativistic Gravity in Space, eds C. Lämmerzahl, C. W. F.Everitt and F. W. Hehl, Vol. 562 of Lecture Notes in Physics (Berlin: Springer Verlag), 310.

Nobili, A. M., Milani, A., and Farinella, P. (1987). Testing Newtonian gravity in space. Phys. Lett. A 120, 437-441. doi: 10.1016/0375-9601(87)90105-8

Nobili, A. M., Milani, A., and Farinella, P. (1988). The orbit of a space laboratory for the measurement of G. Astronom. J. 95, 576-578. doi: 10.1086/ 114657

Nobili, A. M., Milani, A., Polacco, E., Roxburgh, I. W., Barlier, F., Aksnes, K., et al. (1990). The Newton mission - a proposed manmade planetary system in space to measure the gravitational constant. ESA J. 14, 389-408.

Nobili, A. M., and Will, C. M. (1986). The real value of Mercury's perihelion advance. Nature 320, 39-41. doi: 10.1038/320039a0

Paik, H. J. (1989). Tests of general relativity in earth orbit using a superconducting gravity gradiometer. Adv. Space Res. 9, 41-50. doi: 10.1016/02731177(89)90006-9

Paik, H. J. (2008). Detection of the gravitomagnetic field using an orbiting superconducting gravity gradiometer: principle and experimental considerations. Gen. Relat. Gravit. 40, 907-919. doi: 10.1007/s10714-007-0582-4
Petit, G., and Luzum, B. (2010). IERS Conventions (2010). IERS Tech. Note 36:1. Available online at: http://www.iers.org/nn_11216/sid_FC7D9322CF807240D 40C53641833295B/nsc_true/IERS/EN/Publications/TechnicalNotes/tn36.html

Pijpers, F. P. (1998). Helioseismic determination of the solar gravitational quadrupole moment. Month. Notic. R. Astronom. Soc. 297, L76-L80. doi: 10.1046/j.1365-8711.1998.01801.x

Sahni, V., and Shtanov, Y. (2008). Apsis:. an artificial planetary system in space to probe extra-dimensional gravity and MOND. Int. J. Modern Phys. D 17, 453-466. doi: 10.1142/S0218271808012127

Sanders, A. J., Alexeev, A. D., Allison, S. W., Antonov, V., Bronnikov, K. A., Campbell, J. W., et al. (2000). Project SEE (Satellite Energy Exchange): an international effort to develop a space-based mission for precise measurements of gravitation. Class. Q. Grav. 17, 2331-2346. doi: 10.1088/0264-9381/17/ $12 / 305$

Sanders, A. J., Alexeev, A. D., Allison, S. W., Bronnikov, K. A., Campbell, J. W., Cates, M. R., et al. (1999). Project SEE (Satellite Energy Exchange): proposal for space-based gravitational measurements. Measure. Sci. Technol. 10, 514-524. doi: 10.1088/0957-0233/10/6/317

Sanders, A. J., and Deeds, W. E. (1992). Proposed new determination of the gravitational constant G and tests of Newtonian gravitation. Phys. Rev. D 46, 489-504. doi: 10.1103/PhysRevD.46.489

Seidelmann, P. K., Archinal, B. A., A’Hearn, M. F., Conrad, A., Consolmagno, G. J. Hestroffer, D., et al. (2007). Report of the IAU/IAG Working Group on cartographic coordinates and rotational elements: 2006. Celest. Mech. Dyn. Astron. 98, 155-180. doi: 10.1007/s10569-007-9072-y

Smith, D. E., Zuber, M. T., Phillips, R. J., Solomon, S. C., Neumann, G. A., Lemoine, F. G., et al. (2010). The equatorial shape and gravity field of Mercury from MESSENGER flybys 1 and 2. Icarus 209, 88-100. doi: 10.1016/j.icarus.2010.04.007

Soffel, M., Klioner, S. A., Petit, G., Wolf, P., Kopeikin, S. M., Bretagnon, P., et al. (2003). The IAU 2000 resolutions for astrometry, celestial mechanics, and metrology in the relativistic framework: explanatory supplement. Astron. J. 126, 2687-2706. doi: 10.1086/378162

Solomon, S. C., McNutt, R. L., Gold, R. E., and Domingue, D. L. (2007). MESSENGER Mission Overview. Space Sci. Rev. 131, 3-39. doi: 10.1007/s11214007-9247-6

Synge, J. L. (1927). On the Geometry of Dynamics. R. Soc. Lond. Philos. Trans. A 226, 31-106. doi: 10.1098/rsta.1927.0002

Theiss, D. S. (1985). A general relativistic effect of a rotating spherical mass and the possibility of measuring it in a space experiment. Phys. Lett. A 109, 19-22. doi: 10.1016/0375-9601(85)90382-2

Theiss, D. S. (1992). "A gradiometer experiment to detect the gravitomagnetic field of the earth," in Relativistic Gravity Research, Vol. 410 of Lecture Notes in Physics, eds J. Ehlers and G. Schäfer, (Berlin Springer Verlag), 131. doi: 10.1007/3-54056180-3 6

Will, C. M. (2009). The confrontation between general relativity and experiment. Space Sci. Rev. 148, 3-13. doi: 10.1007/s11214-009-9541-6

Will, C. M. (2014). Incorporating post-Newtonian effects in N-body dynamics. Phys. Rev. D 89:044043. doi: 10.1103/PhysRevD.89.044043

Conflict of Interest Statement: The author declares that the research was conducted in the absence of any commercial or financial relationships that could be construed as a potential conflict of interest.

Received: 08 June 2014; paper pending published: 10 July 2014; accepted: 24 July 2014; published online: 14 August 2014.

Citation: Iorio L (2014) Orbital motions as gradiometers for post-Newtonian tidal effects. Front. Astron. Space Sci. 1:3. doi: 10.3389/fspas.2014.00003

This article was submitted to Cosmology, a section of the journal Frontiers in Astronomy and Space Sciences.

Copyright (c) 2014 Iorio. This is an open-access article distributed under the terms of the Creative Commons Attribution License (CC BY). The use, distribution or reproduction in other forums is permitted, provided the original author(s) or licensor are credited and that the original publication in this journal is cited, in accordance with accepted academic practice. No use, distribution or reproduction is permitted which does not comply with these terms. 